\title{
Design of Kicker/Bumper Magnet and PFN for PAR
}

\author{
By JU WANG AND GERALD J. VOLK*
}

Introduction

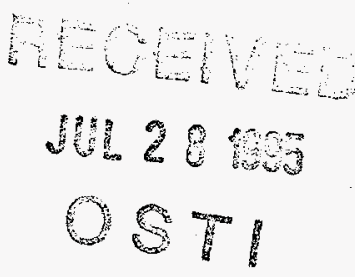

Three fast pulsed kicker/bumper magnets are required in the positron accumulator ring (PAR) for the purpose of beam injection and/or extraction at $450 \mathrm{MeV}$. According to the Conceptual Design Report (CDR), these three magnets have identical specifications and are expected to produce identical magnetic fields. Therefore, they will have the same design. Each kicker/bumper magnet is required to generate a magnetic field of $0.06 \mathrm{~T}$ with a rise time of 65 ns, a flat top of $80 \mathrm{~ns}$ and a fall time of $90 \mathrm{~ns}$, respectively.

A fast pulsed magnet system normally consists of a high voltage dc power supply, charging/discharging switches which are tetrodes and thyratrons in most cases, a pulse forming network (PFN), a magnet assembly consisting of a ferrite magnet, a matching capacitor or capacitors and a load (or termination) resistor. The primary objective of this study is the design of the pulse forming network and magnet assembly.

\section{Pulse Forming Network}

The function of a pulse forming network is to generate a current of certain desired waveform when the discharging switch is closed. As the current travels through a magnet it produces a magnetic field inside the air gap of the magnet. If the magnet is not saturated the magnetic field has the same shape as the current waveform.

There are many different circuit configurations used in pulse forming networks. The simplest one is to use coaxial cables (transmission lines) as the PFNs. In this case the coaxial

\footnotetext{
${ }^{*}$ Gerald J. Volk is with the Intense Pulsed Neutron Source.
} 



\section{DISCLAIMER}

Portions of this document may be illegible in electronic image products. Images are produced from the best available original document. 
cables are charged to a voltage that is equal to twice the peak pulse current times the resistance of the load (or termination) resistor. When the switch between the cables and the load, which is usually a resistor with its resistance matching the cable impedance, is closed, one half of the cable voltage drops on the load resistor and produces a current in the load. The current propagates from the load to the other end of the cables where a complete reflection of the current will occur. When the reflected current travels back to the load, all the energy stored in the cables is dissipated and the current becomes zero. For this kind of PFN, the duration of the pulse waveform is equal to twice the cable length divided by the propagation velocity. It is usually used for pulses with durations under $100 \mathrm{~ns}$.

The second way is to use equal inductors and capacitors to construct PFNs (as shown in Figure 1). This type of PFNs is actually lumped equivalents to coaxial cables and, hence, behaves similarly to coaxial cables. They are normally used to generate pulses with durations over several hundred nanoseconds.

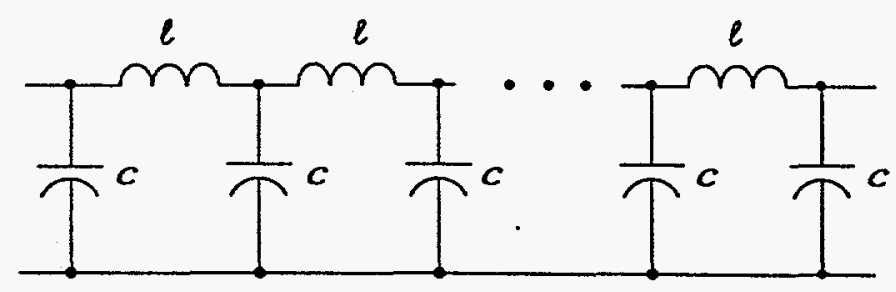

Figure 1. Equal L-C pulse forming network

Another type of PFNs is called type-C Guillemin network. For type-C Guillemin network, Fourier analysis is used to calculate the frequency components of the desired pulse waveform. Then multiple L-C branches are connected in parallel with each $\mathrm{L}-\mathrm{C}$ branch generating one frequency component. The number of the L-C branches depends on the requirement of the waveform. When the requirement on the flatness of the flat top of the pulse is strict this method becomes practically unsuitable because too many L-C branches would be 
needed to reduce the ripples in the flat top. There are other types of Guillemin networks. Some of them are the different variations of type-C network.

Among many types of PFNs, the best candidates for the PAR kicker/bumper are coaxial cables and the equal L-C network. In the design, a combination of coaxial cables and equal L-C network is used. The cable section of the PFN consists of two 12 meter paralleled cables for each half turn coil. The cable impedance is approximately $13 \Omega$, the inductance is $101.7 \mathrm{nH} / \mathrm{m}$ and the capacitance is $377.3 \mathrm{pF} / \mathrm{m}$. With these parameters, the propagation velocity of the voltage and current in the cable is $0.161 \mathrm{~m} / \mathrm{ns}$. The equal L-C section of the PFN is made of the magnet and the matching capacitors. Since a capacitor or capacitors have to be added to the magnet to match the cable impedance in order to minimize the reflections between the cables and the magnet, it will be advantageous if the magnet can contribute to the pulse forming network. With the capacitors matching the above cables, the magnet is equivalent to two 4.15 meter paralleled cables. It will take $25.7 \mathrm{~ns}$ for the current and voltage to pass through the magnet.

\section{Magnet}

In the CDR a window-frame magnet was proposed. This magnet is $35 \mathrm{~cm}$ long and has an air gap of $11 \mathrm{~cm}$ by $5.3 \mathrm{~cm}$. The air gap size is determined by the sizes of the vacuum chamber and the copper conductors with their insulations. The cross section of the magnet is shown in Figure 2.

Assuming that the magnetic field inside the air gap is uniform, the magnetic field intensity inside the ferrites is negligible compared with that in the air gap, and that the end effect can be ignored, the magnetic field intensity, $\mathbf{H}$, in the air gap can be calculated with Ampere's circuital law 


$$
\mathbf{H}=\frac{I}{g}
$$

where $I$ is the current in the coil and $g$ is the length of the air gap. Then, the flux density, B, in the air gap is given as

$$
\mathbf{B}=\frac{\mu_{0} I}{g}
$$

When $\mathbf{B}$ is available, the current required to produce $\mathbf{B}$ can be obtained using

$$
I=\frac{\mathrm{B} g}{\mu_{0}}
$$

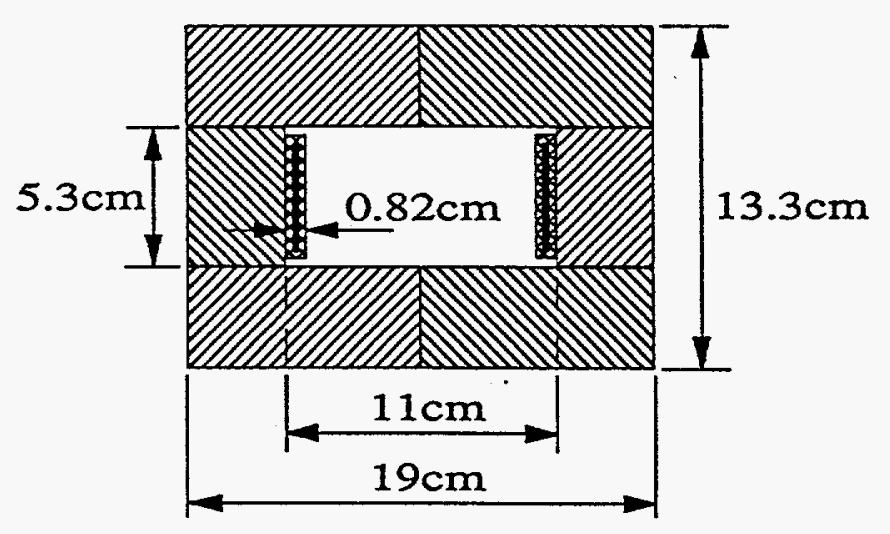

Figure 2. Cross section of the PAR kicker/bumper magnet

For the magnet in Figure 2, to generate a magnetic field of $0.06 \mathrm{~T}$, the required current will be $2530.56 \mathrm{~A}$.

The inductance of the coil can be calculated as 


$$
L=\frac{\mu_{0} w l}{g}
$$

where $w$ is the width of the air gap (the distance between the centers of two conductors) and $l$ is the length of the magnet. For the given geometry of the magnet, the inductance $L$ is equal to $844.8 \mathrm{nH}$.

Since the rise time of the current, hence the rise time of the magnetic field, mostly depends on the magnitude of the inductance, the inductance of the magnet has to be as small as possible. The total inductance of the magnet is fixed for the given geometry of the magnet: The only way to reduce the inductance is to divide the one single turn coil into two half turn coils. In doing so, the inductance seen by the cables is reduced to one half. However, the number of the required cables is increased by a factor of two.

The magnet will be constructed with ferrite CMD5005, by Ceramic Magnetics, Inc. The thickness of the ferrite blocks is selected as $4 \mathrm{~cm}$. This results in a magnetic field of about 764 Gauss inside the ferrites, below its saturation field. Thin copper films will be inserted into the top and the bottom ferrites to completely decouple two half turn coils.

\section{Impedance Match of the Magnet and the Coaxial Cables}

In general, there are reflections at the connection point of two different circuit components when the voltage and current travel from one component to the other unless the two components have matched impedance. Reflections may cause overvoltage in the circuit and damage the circuit components. Reflections can also distort current waveforms which will in turn distort magnetic fields. Therefore, reflections should be eliminated or minimized when elimination is impossible. Since the magnet will be connected to the coaxial cables and act as part of the pulse forming network, it has to have an impedance that matches the cable impedance. 
To match the cables, the ideal way is to build a magnet with a coaxial structure. However, the size of the required aperture for the beam is relatively large $(11 \mathrm{~cm}$ by $5.3 \mathrm{~cm})$ and the cable impedance is relatively small ( $6.5 \Omega$ for two paralleled cables), so it is extremely difficult, if not impossible, to construct a coaxial magnet that has a large air gap and a small impedance. Therefore a window-frame magnet has been chosen and a lumped capacitor or capacitors will be added to the magnet to match the cable impedance. Since the magnet is a lumped element, to better match the cables, the magnet can be divided longitudinally into several sections with multiple capacitors. Ideally, the more sections the magnet is divided into, the better it matches the cable. However, the number of the sections cannot be very large due to the limitation of the physical sizes of the magnet and the capacitors.

Now that the magnet is divided by the capacitors, it can be part of the pulse forming network and is precharged to the PFN voltage. When the discharge switch (thyratron) is closed, the current will travel through the magnet back to the input of the PFN. The minimum rise time of the current in the termination resistor is equal to

$$
T_{r, t e r m}=\frac{\pi \sqrt{L_{1 / 2} C}}{2 \mathrm{n}}
$$

where $L_{1 / 2}$ is the half turn magnet inductance, $C$ is the total capacitance of the capacitors added to one half of the magnet and $\mathrm{n}$ is the number of the capacitors. $C$ can be calculated as

$$
C=\frac{\mathrm{k}^{2} L_{12}}{L_{\text {cable }}} C_{\text {cable }}
$$

where $C_{\text {cable }}$ and $L_{\text {cable }}$ are the cable capacitance and inductance per unit length, and $\mathrm{k}$ is the number of cables in parallel. In the design of the PFN, two cables are connected in parallel, so $\mathrm{k}$ $=2$. With the given cable and magnet parameters, a total capacitance of $6.26 \mathrm{nF}$ will be 
required. Because the limitation of the physical sizes of the magnet and the capacitors, only four capacitors can be connected to each half of the magnet. Each capacitor is $1.56 \mathrm{nF}$ and each section of the magnet is $105.6 \mathrm{nH}$.

Substituting $L_{1 / 2}, C$ and the number of capacitors into equation (5) gives the minimum rise time, $T_{r, t e r m}$, of the current in the termination resistor as $20.2 \mathrm{~ns}$. The minimum rise time of the magnetic field of the whole magnet will be the sum of $T_{r, t e r m}$ and the time required for the current to pass through the magnet, and will be 45.9 ns.

When multiple capacitors are connected to the magnet, there may exist voltage and current oscillations between the capacitors and the magnet. The computer simulation has shown that without any damping, the oscillations destroy the flat top of the magnetic field. Hence, damping resistors have to be connected in series with the capacitors. But, the damping resistors slow down the rise time of the current, especially if the circuit is critically damped. Because the damping resistors are unavoidable, the compromise has to be made between the rise time and the flat top. According to the computer simulations, the best result is obtained when the circuit is slightly under damped and the corresponding resistance of the damping resistors is $6.5 \Omega$.

A basic circuit diagram of the kicker/bumper unit is shown in Figure 3. The circuit diagram shows that the cathode of the thyratron is connected to the negative pole of the high voltage dc supply which will be connected to ground. In this way isolation transformers will not be necessary for the trigger control unit, the cathode heater or the reservoir heater of the thyratron.

\section{Control of the Charging and Discharging of the PFN}

The first and the second kicker magnets will be operated at $60 \mathrm{~Hz}$ for injection. The third kicker magnet will be operated at $2 \mathrm{~Hz}$ for extraction. To control the charging and discharging 


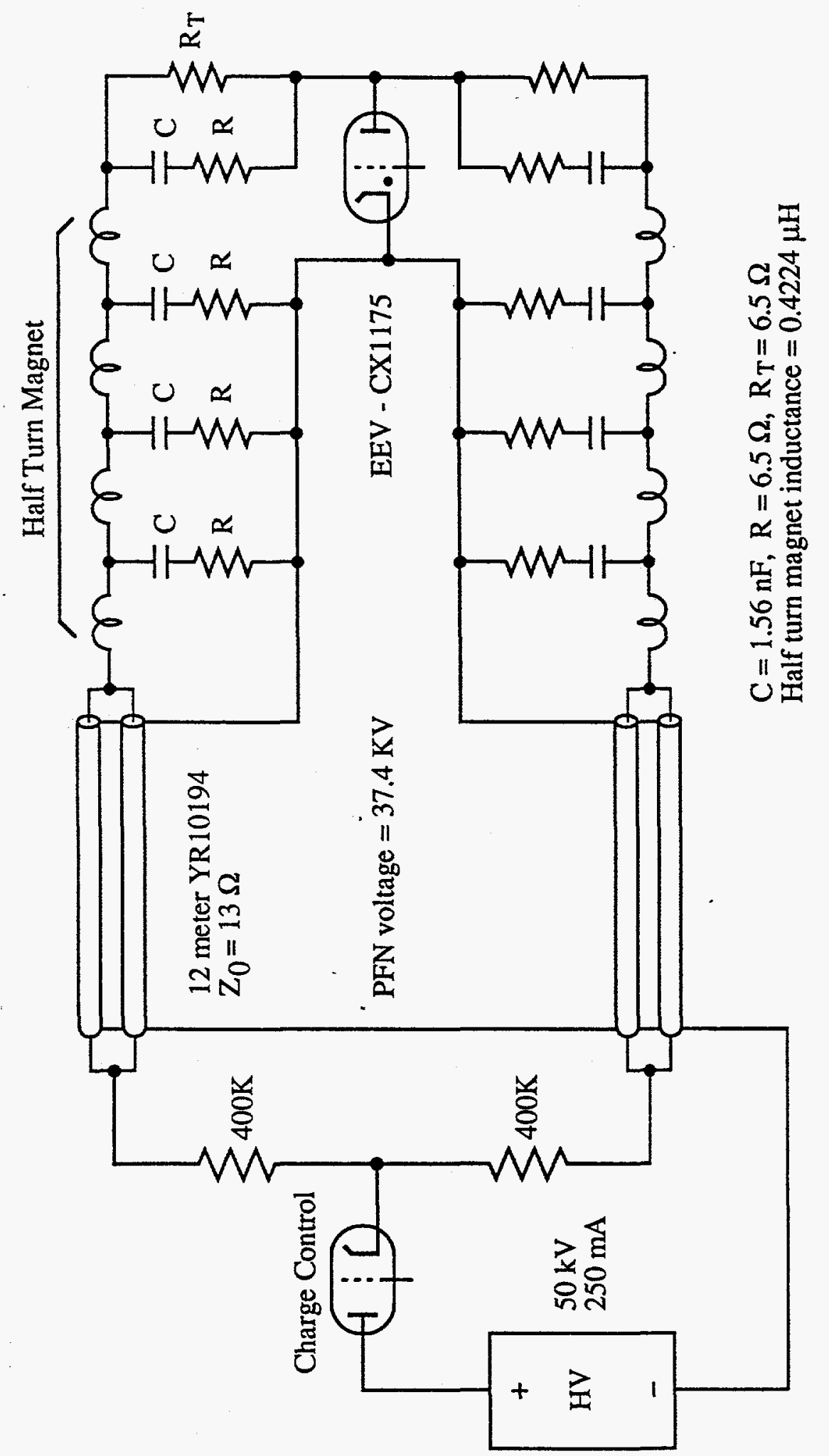

 
of the pulse forming network, two control (clock) signals will be required from the central computer for triggering the thyratrons and the tetrodes.

Since the signal for triggering the thyratrons to discharge the PFNs is the most critical in terms of timing, it should be used as the time reference. The signal is also sent to the control circuit to open the tetrodes, should they be still closed, to ensure that the thyratrons will be able to open after all the energy in the PFNs is dissipated.

The time jitter of the magnetic field has to be less than 2 ns. Most part of the jitter is expected to come from the thyratron, typically $1 \mathrm{~ns}$, and the triggering circuit, $0.5 \mathrm{~ns}$ approximately. So, the jitter in the trigger signal for the thyratrons from the central computer has to be less than $0.5 \mathrm{~ns}$.

The power supplies and the charging circuits are designed such that it will take $14 \mathrm{~ms}$ to charge the PFNs to the required voltage. After the PFN voltage reaches the required voltage, a feedback signal from the measuring circuit turns off the tetrodes. The PFNs should not be left too long before they are discharged since the voltage will drop due to the leakage of the PFNs. The charging signal from the central computer is required at least $14 \mathrm{~ms}$ plus a few $\mu \mathrm{s}$ for the time jitter prior to the discharging signal.

\section{Computer Simulation of the Circuit}

The circuit shown in Figure 3 has been simulated with PSpice program, by MicroSim Corporation, Irvine, CA. In the simulation only one half-turn magnet and the associated cables were simulated. The $12 \mathrm{~m}$ coaxial cables were represented by 30 series connected identical $\pi$ type L-C subcircuits. Two different cases were considered for the cables. One assumed that the cables were lossless. The other took consideration of the copper losses of the cables. In the case 
of the copper losses, the skin effect was taken into account by using equation (7) to estimate the resistance.

$$
r=8.3(f)^{1 / 2}\left(\frac{1}{d}+\frac{1}{D}\right) \quad \mu \Omega / \mathrm{m}
$$

In equation (7), $f$ is the frequency in $\mathrm{Hz}, d$ is the cable diameter at the inner conductor and $D$ is the cable diameter at the outer conductor. Dimensions for $d$ and $D$ are in centimeters. According to the required pulse width, $f$ is about $4 \mathrm{MHz}$. Diameters $d$ and $D$ are 3.64 and 2.25 $\mathrm{cm}$, respectively. The estimated cable resistance is $0.0119 \Omega / \mathrm{m}$, about five times the dc resistance.

The simulations show no significant differences in the rise time and the flat top for two different cases, with and without copper losses. The losses in the dielectrics could not be included in the simulation because no data are available.

The thyratron was represented by a $100 \mathrm{~V}$ dc voltage source and a $20 \mathrm{nH}$ inductor (suggested by the manufacturer). This may have over simplified the turn-on process of the thyratron. In reality, the turn-on process may take a longer time than that due to a $20 \mathrm{nH}$ inductor. If a longer turn-on process does happen, a magnetic switch (saturable magnet) can be added to the anode (or cathode) of the thyratron. This technique can significantly reduce the turn-on time. Furthermore, it may also extend the life time of the thyratron.

The simulations were also done for the circuit with only one matching capacitor for each half magnet, and the circuit with the magnet divided by multiple capacitors but no damping resistors, so that different designs could be compared. The comparison shows that the circuit shown in Figure 3 produces the best current waveform.

Figures 4 through 10 show part of the simulation results. 


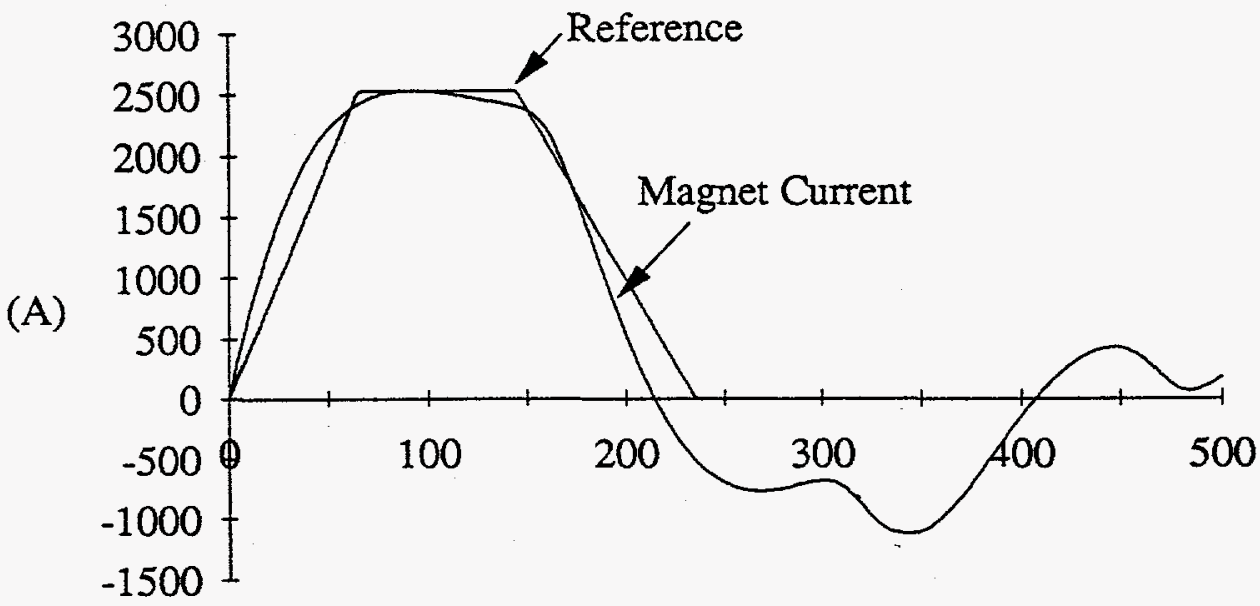

TIME (ns)

Figure 4. Simulation result for the magnet with only one matching capacitor.

(A)

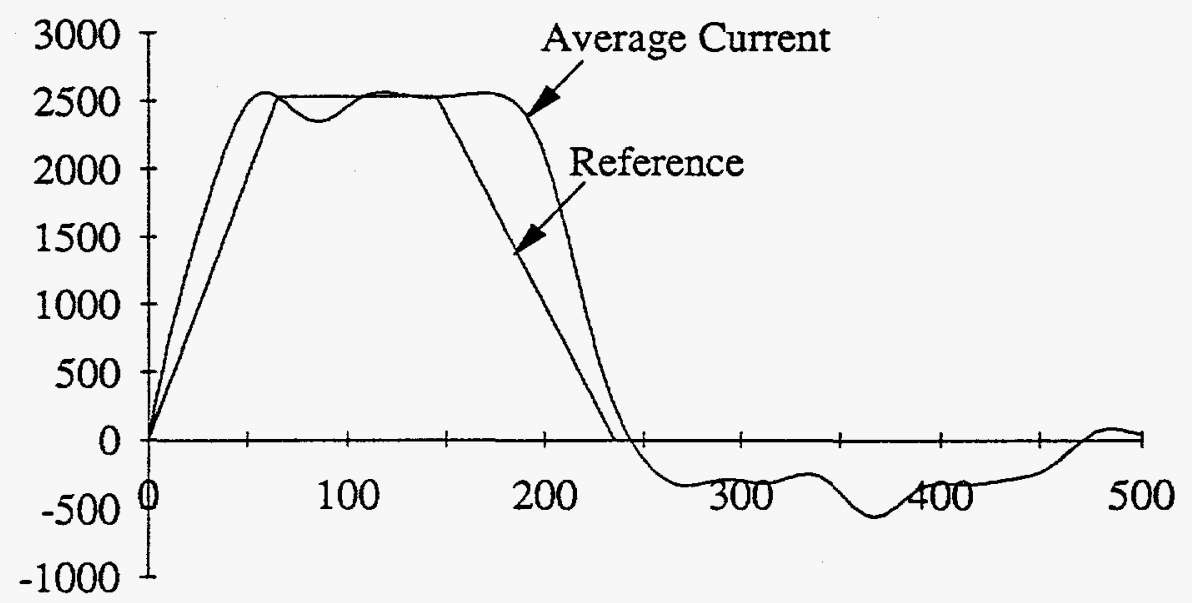

TIME (ns)

Figure 5. Simulation result for the magnet without damping resistors. 


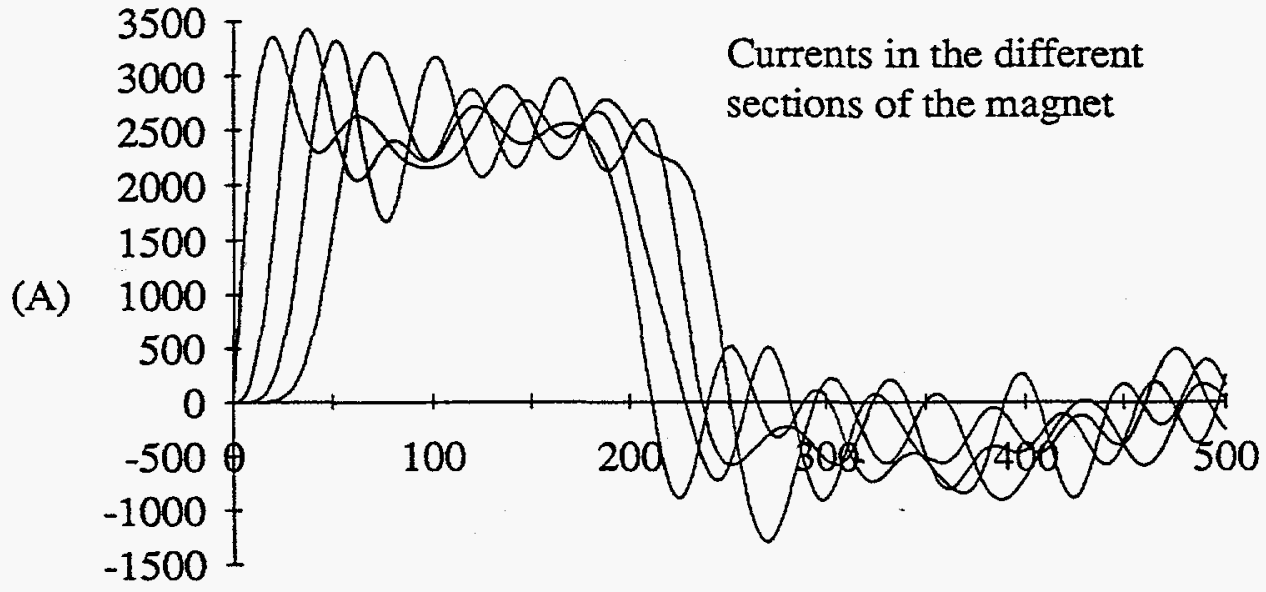

TIME (ns)

Figure 6. Simulation result for the magnet without damping resistors.

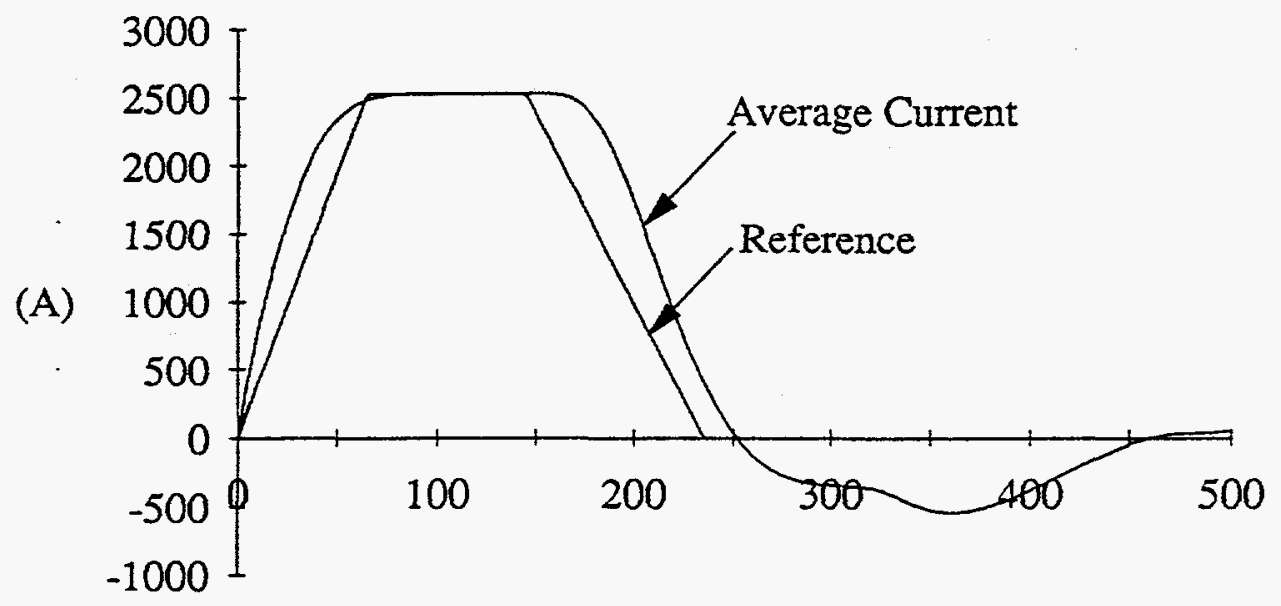

TIME (ns)

Figure 7. Simulation result for the circuit in Figure 3. 


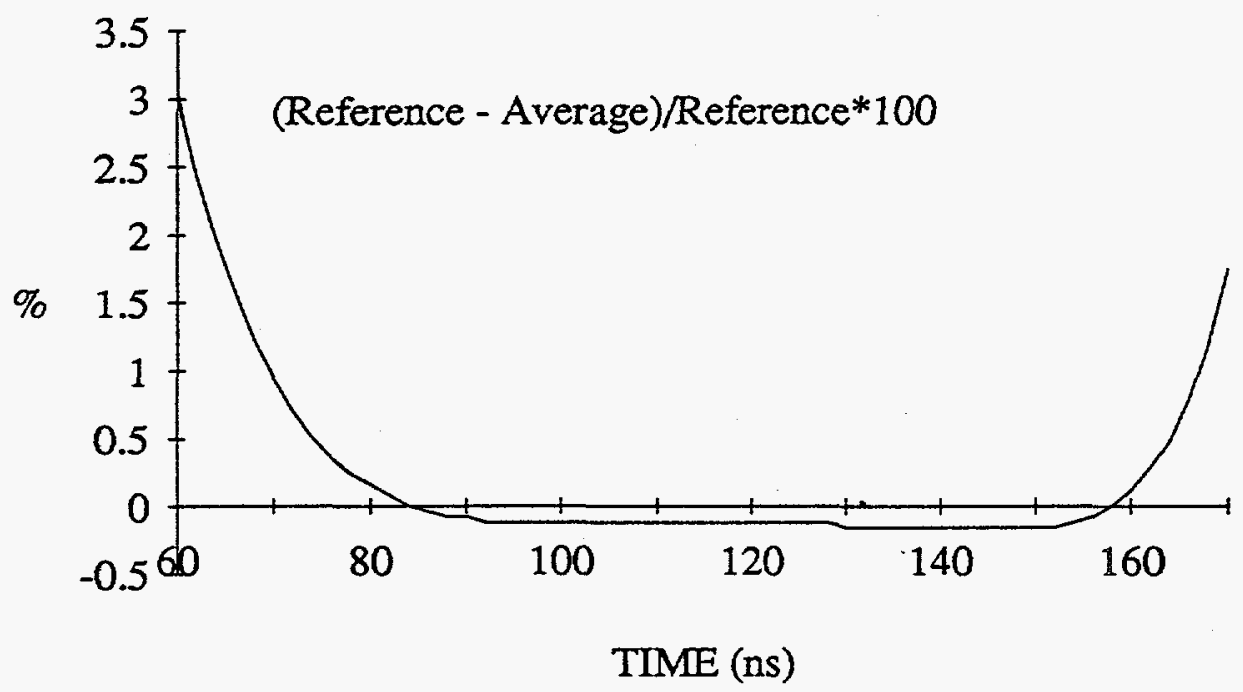

Figure 8. Simulation result for the circuit in Figure 3.

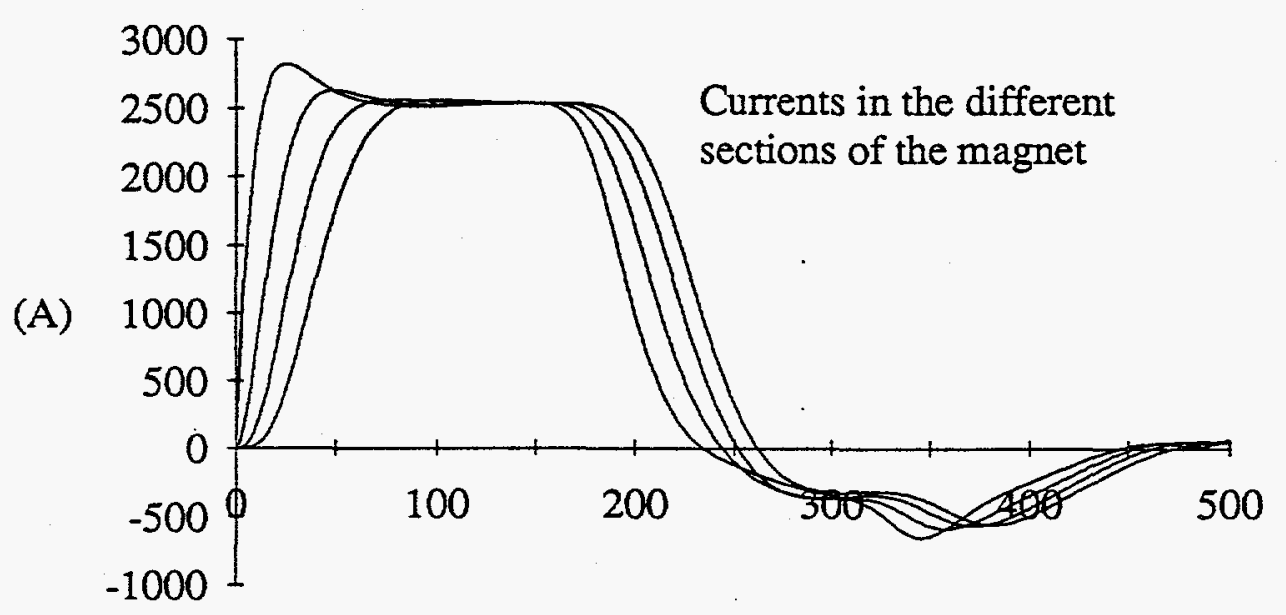

TIME (ns)

Figure 9. Simulation result for the circuit in Figure 3. 
(A)

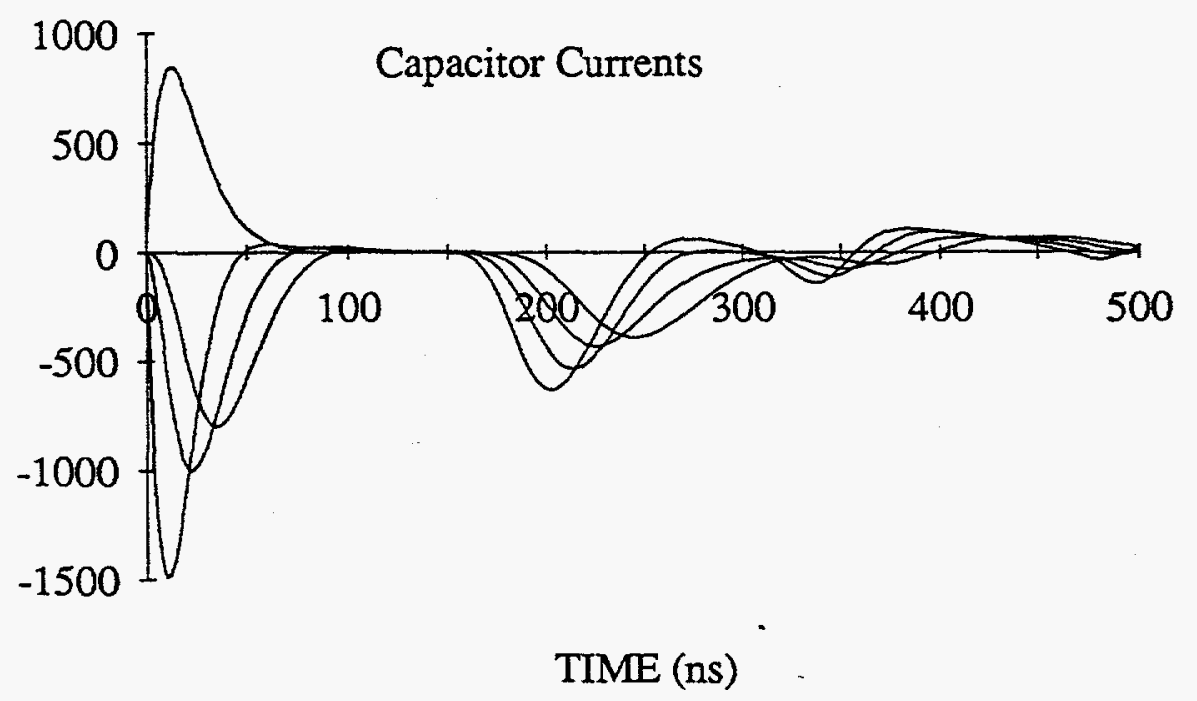

Figure 10. Simulation result for the circuit in Figure 3.

\section{Reference}

1. Sarjeant, James W. and Dollinger, R. E., "High-Power Electronics", TAB BOOKS Inc., 1989.

2. Bulos, F. et al, "Some Fast Beam Kicker Magnet Systems at SLAC", CH23879/87/0000-1884\$1.00 @ IEEE.

3. Bartelson, C. et al, "Kicker for the SLC Electron Damping Ring", CH23879/87/0000-1582 \$1.00 @ IEEE.

4. Ducimetière, L. and Fiander, D. C., "Commutation Losses of a Multigap High Voltage Thyratron", 19th Power Modulator Symposium, San Diego, CA, June 26-28, 1990.

5. Rust, Ken and McDuff, Glen, "Life Extension of Thyratrons in Short Pulse Circuits with the Use of Saturable Magnetic Sharpeners", 19th Power Modulator Symposium, San Diego, CA, June 26-28, 1990.

6. Giacoletto, L. J., "Electronics Designers' Handbook”, Second Edition, McGraw-Hill Book Company. 